Applications of summation-by-parts

\title{
operators
}

Oskar Ålund 

Linköping Studies in Science and Technology.

Dissertations, No. 2106

\title{
Applications of summation-by-parts operators
}

\author{
Oskar Ålund
}

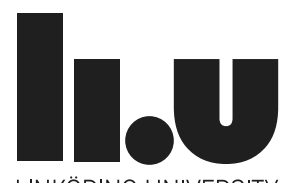

LINKÖPING UNIVERSITY

Department of Mathematics, Division of Computational Mathematics

Linköping University, SE-581 83 Linköping, Sweden

Linköping 2020 
(oc) EY-Ne This work is licensed under a Creative Commons AttributionNonCommercial 4.0 International License.

https://creativecommons.org/licenses/by-nc/4.0/

Linköping Studies in Science and Technology. Dissertations, No. 2106

\section{Applications of summation-by-parts operators}

Copyright (C) Oskar Ålund, 2020

Division of Computational Mathematics

Department of Mathematics

Linköping University

SE-581 83, Linköping, Sweden

oskar.alund@liu.se

www.liu.se/mai/ms

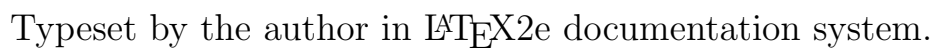

ISSN 0345-7524

ISBN 978-91-7929-753-4

Printed by LiU-Tryck, Linköping, Sweden 2020 


\section{Abstract}

Numerical solvers of initial boundary value problems will exhibit instabilities and loss of accuracy unless carefully designed. The key property that leads to convergence is stability, which this thesis primarily deals with. By employing discrete differential operators satisfying a so called summationby-parts property, it is possible to prove stability in a systematic manner by mimicking the continuous analysis if the energy has a bound. The articles included in the thesis all aim to solve the problem of ensuring stability of a numerical scheme in some context. This includes a domain decomposition procedure, a non-conforming grid coupling procedure, an application in high energy physics, and two methods at the intersection of machine learning and summation-by-parts theory. 



\section{Sammanfattning på svenska}

Med viss frustration accepterar vi att vårt universum inte avslöjar sina intentioner med någon särskild tydlighet. Eller så är vi inte tillräckligt perceptiva. Hur som helst så visar det sig att de mest sofistikerade verktyg vi har för att beskriva fysikaliska förlopp, så kallade partiella differentialekvationer, ofta saknar prediktiva egenskaper per se. Istället beskriver de ett precist samband mellan nu och snart. De fångar på ett elegant sätt universums ebb och flod (både bokstavligt och bildligt talat), och beskriver hennes ständiga föränderlighet i termer av hennes nuvarande tillstånd. Tyvärr är svaret på frågan "Idag skiner solen, men hur blir vädret nästa torsdag?" inte enkelt funnet bland väderekvationerna, med otaliga förstörda semestrar som konsekvens. Ekvationerna må vara en perfekt beskrivning av vädret. Kanske har de till och med ett och endast ett möjligt väderförlopp. Trots detta, i vad som framstår som ett slags gudomligt "practical joke", så är vi intelligenta nog att formulera ekvationerna, men inte intelligenta nog att lösa dem. Istället måste vi förlita oss på datorer och approximativa lösningar.

Approximativa lösningar beräknas genom att differentialekvationen omformuleras på ett sätt som är hanterbart för datorer, en så kallad diskretisering. Detta görs genom att på en ändlig beräkningsdomän generera ett $n \ddot{a} t$, och formulera en approximativ variant av differentialekvationen på detta nät. Diskretiseringsprocessen är fylld med fallgropar. Oförsiktighet i detta skedet kan leda till opålitliga beräkningsresultat. I bästa fall är det uppenbart att något har gått fel, men i värsta fall ser resultatet rimligt ut fastän det egentligen är helt fel. Därför är det helt nödvändigt att teoretiskt visa att en diskretisering faktiskt genererar en approximativ lösning till ursprungsekvationen. Inom beräkningsmatematiken kallar vi denna efterssträvansvärda egenskap konvergens.

En relaterad och nödvändig förutsättning för konvergens är stabilitet. I denna avhandling använder vi så kallade summation-by-parts operatorer för 
att säkerställa stabilitet för att antal olika diskretiseringar. Dessa operatorer används inom en mängd olika diskretiseringsmetoder, såsom finita differenser, finit volym, och vissa Galerkinmetoder. Avhandlingen omfattar fem artiklar. Artikel I beskriver en metod där beräkningsområdet delas upp i mindre bitar för att öka beräkningseffektiveteten. Optimal uppdelning undersöks och stabilitet visas. Artikel II behandlar kurvlinjära nät och stabila icke-konforma kopplingar mellan olika nät. I Artikel III utvecklar vi en ny typ av invariansbevarande diskretisering av Lindblads ekvation. Artikel IV beskriver hur neurala nätverk kan användas som stöd för beräkningar av så kallade gränsskikt. Slutligen studerar vi i Artikel V hur idéer och verktyg från maskininlärning kan användas för att konstruera diskretiseringar på överlappande nät. 


\section{Acknowledgments}

I would like to thank my supervisor Jan Nordström for his continuous support and insight. Your enthusiasm leaves no room for procrastination and your experience has been invaluable.

Without mathematics life is senseless, but without love it is meaningless. To my parents, the most inspiring people I know, I am forever grateful. You taught me empathy, respect, humility, joy, gratitude, and stoicism. Your unconditional love has carried me from an anxious childhood to a genuinely happy adulthood. I love you.

I started this journey at a dismal time in my life, and to everyone who helped turn it all around when I moved to Linköping, thank you from the bottom of my heart. I miss our evenings at Pitchers. Thank you Roghi for being the way you are. You make the world a better place. Thank you Nancy for being an amazing friend. Thank you also to Fredrik (the guy I work with), his mother, and Matilda. You guys are the best.

Finally, Bana, thank you for being you. You are my moon and the bravest person I know. Let us grow old together. 



\section{List of Papers}

I Ålund, Oskar, and Jan Nordström. "A Stable Domain Decomposition Technique for Advection-Diffusion Problems." Journal of Scientific Computing 77.2 (2018): 755-774.

II Ålund, Oskar, and Jan Nordström. "Encapsulated high order difference operators on curvilinear non-conforming grids." Journal of Computational Physics 385 (2019): 209-224.

III Ålund, Oskar, Yukinao Akamatsu, Fredrik Laurén, Takahiro Miura, Jan Nordström, and Alexander Rothkopf. "Trace preserving quantum dynamics using a novel reparametrization-neutral summation-by-parts difference operator." Accepted in Journal of Computational Physics (2020).

IV Nordström, Jan, and Oskar Ålund. "Neural Network Enhanced Computations on Coarse Grids." Journal of Computational Physics 425 (2020)

V Ålund, Oskar, Gianluca Iaccarino, and Jan Nordström. "Learning to Differentiate." Journal of Computational Physics 424 (2020).

I derived most of the theory and numerical results presented in paper I, II, IV, and V with editorial support from my supervisor Jan Nordström (as well as from Gianluca Iaccarino in the case of paper V). In paper III, I developed the spatial discretization in collaboration with Fredrik Lauren and Jan Nordström. 



\section{Contents}

Abstract

Sammanfattning på svenska $\quad$ iii

Acknowledgments $\quad$ v

List of Papers vii

1 Introduction 1

2 Initial boundary value problems 3

3 Summation-by-parts operators $\quad 9$

4 Weak boundary conditions 13

$\begin{array}{lll}5 & \text { Artifical neural networks } & 17\end{array}$

6 Summary of articles $\quad 21$

6.1 Article I . . . . . . . . . . . . . . . . . . . . . 21

6.2 Article II . . . . . . . . . . . . . . . . . . . . . . . . . . . . 23

6.3 Article III . . . . . . . . . . . . . . . . . . . . . . . . . . . . 24

6.4 Article IV . . . . . . . . . . . . . . . . . . . . . . 26

6.5 Article V...................... . . 26

$\begin{array}{ll}\text { References } & 29\end{array}$ 



\section{Introduction}

Partial Differential Equations (PDEs) are among of the most powerful tools used in mathematical modelling. These equations permeate virtually all predictive sciences, and are used to model everything from subatomic interactions [1] to human scale phenomena [2] to galactic evolution [3]. In essence, a $\mathrm{PDE}$ is an explicit relation between one or several quantities and their rates of change (potentially both temporal an spatial). Such relations often arise from first principles such as mass and energy conservation, or Newton's laws of motion, together with some clever mathematics. Sadly, these equations are rarely easy (or even possible) to solve analytically. In fact, even the existence of a solution to a PDE is not guaranteed in general. Perhaps the most famous unsolved existence problem is that of the Navier-Stokes equations, describing fluid flow [4]. The advent of computers, while not solving the problem of finding analytical solutions, has made it possibe to "solve" PDEs in a more practical sense; namely approximately.

Approximate solutions are computed by reformulating the differential equation in a way that is managable by computers; a so called discretization. This is done by considering a bounded computational domain on which a finite mesh or grid is introduced. The process of discretizing a differential equation is filled with pitfalls. Carelessness in this phase may lead to unreliable results. In the best case it is obvious that something failed, but in the worst case the results appear plausible but are in fact wrong. Therefore it is necessary to build discretizations based on firm theoretical foundations, so that one can be certain that an approximate solution to the original equation is computed. In computational science this desirable property is known as convergence.

A related and necessary condition for convergence is stability. In this thesis we apply so called summation-by-parts operators to ensure stability 
for a number of different discretizations. Summation-by-parts operators include a wide range of discretization methods, such as finite differences, finite volume, and discontinuous Galerkin methods. The thesis comprises five articles. Article I describes a method where the computational domain is split into smaller parts in order to increase computational efficiency. Article II deals with curvilinear grids and stable non-conforming couplings between grids. In article III we develop a novel trace preserving discretization for the Lindblad equation, describing the evolution of dissipative quantum systems. Article IV describes how artificial neural networks can be used to support computations of boundary layers. Finally, in article $\mathrm{V}$ we study how ideas and tools from machine learning may be used to construct discretizations on overlapping grids.

Brief summaries of the articles and novel results will be outlined after the next few sections which serve as an introduction to the topics of initial boundary value problems, weak boundary conditions, summation-by-parts operators, and artificial neural networks. 


\section{Initial boundary value problems}

Suppose we are interested in the behavior of some physical quantity (or set of related quantities) inside a particular region of space. Perhaps we want to study temperature changes in an object, or the flow of a fluid. A common way to describe how a physical quantity behaves is to derive a partial differential equation (PDE) from first principles (e.g. Newtonian or thermodynamic laws). For example, consider a domain $\Omega$ of a d-dimensional space $(d=1,2,3)$, and a conserved scalar quantity $u=u(x, t)$. Suppose that throughout the domain $\Omega$, the flux $F$ of $u$ is known. In other words, there is a vector field $F$ which tells us the rate of flow of $u$ per unit area through a surface at any point of the region. More precisely, if $n$ is the unit normal of a surface, then $F \cdot n$ is the rate of flow through the surface per unit area. In particular, for any subset $A \subset \Omega$, the rate of change in the amount of $u$ inside $A$ is equal to the net inflow through the boundary $\partial A$ :

$$
\frac{d}{d t} \int_{A} u d x=-\int_{\partial A} F \cdot n d s .
$$

Equivalently, by the divergence theorem,

$$
\frac{d}{d t} \int_{A} u d x=-\int_{A} \nabla \cdot F d x .
$$

Since $A$ was chosen arbitrarily, it follows that

$$
u_{t}+\nabla \cdot F=0 \text { for all } x \in \Omega .
$$

Equation (2.1) is known as a conservation law and the flux $F$ typically depends on $u$ in some fashion, either linearly or nonlinearly. It should be noted that equation (2.1) does not alone determine $u$. Two major pieces of additional knowledge of $u$ is necessary: 
1. The values of $u$ in $\Omega$ at some point in time, usually called the initial time, or $t=0$. This piece of information is known as an initial condition.

2. Some appropriate information about $u$ at the boundary $\partial \Omega$ (what appropriate means here is one of the deeper, and in many cases unresolved, questions posed within the theory of partial differential equations). This piece of information is known as a boundary condition.

A PDE such as equation (2.1), together with an initial condition and an appropriate boundary condition, forms an Initial Boundary Value Problem (IBVP). The simplest nontrivial IBVP is obtained by setting the dimension $d=1$, the domain $\Omega=(0,1)$ (the unit interval), and the flux $F=u$ :

$$
u_{t}+u_{x}=0 \text { for all } x \in(0,1) \text { and } t>0 .
$$

Equation (2.2) is known as a linear advection equation. We may intuit from the form of the flux that equation (2.2) describes a constant propagation of $u$ from left to right. After all, at any given point $x \in(0,1)$, the rate of flow in the $x$-direction is equal to $u$. Indeed, if the initial state of $u$ is some function $f$ (in other words, $u(x, 0)=f(x)$ for all $x \in \Omega$ ) and for $t>0$ we have $u(x, t)=f(x-t)$, then

$u_{t}+u_{x}=f^{\prime}(x-t) \frac{d(x-t)}{d t}+f^{\prime}(x-t) \frac{d(x-t)}{d x}=-f^{\prime}(x-t)+f^{\prime}(x-t)=0$.

Hence, $u(x, t)=f(x-t)$ solves the PDE.

But what happens at the left boundary? The attentive reader may have realized that $f$ need not be defined outside $\Omega$, so what does " $f(x-t)$ " mean if $x-t \notin \Omega$ ? If equation (2.2) describes a propagation from left to right, then something must be entering the domain at its left boundary - but what is it? This is where the boundary condition comes in. For the problem to make sense, we have to prescribe what flows into $\Omega$ through the left boundary. More precisely, we add the boundary condition $u(0, t)=g(t)$, where $g$ is some function of time. Note that this issue does not arise at the right boundary. We are perfectly content with allowing information to flow out of the domain. The functions $f$ and $g$ are known as data, because they contain information needed to determine $u$.

Up to this point we have relied on intuition in order to decide what kind of extra information about $u$ that is reasonable to request. Let us try to 
Initial boundary value problems

make things a bit more stringent. In a strictly mathematical sense, what we would like is for the extra conditions to be such that when combined with equation (2.2), the problem uniquely determines $u$. This desire is usually split into two subproblems: the problem of existence and the problem of uniqueness. Books have been written about these problems for various classes of PDEs and we shall not delve deep into them in this thesis. Instead we shift focus to a more subtle but, for computational purposes, painfully relevant problem. The problem of energy boundedness. Formally we require that for any time $T>0$,

$$
\|u(T)\|^{2} \leq K\left(\|f\|^{2}+\|g\|^{2}\right),
$$

where $K$ is bounded for all finite times. The norms in (2.3) are typically $L^{2}$ norms. Relation (2.3) is called an energy estimate. For linear problems (like the advection equation above), an energy estimate of the sort (2.3) implies that the solution varies continuously with data. Indeed, if $v$ satisfies the same linear IBVP as $u$ but with perturbed data $f+\delta f$ and $g+\delta g$, then $v-u$ satisfies the IBVP with the data $\delta f$ and $\delta g$. Hence,

$$
\|v(T)-u(T)\|^{2} \leq K\left(\|\delta f\|^{2}+\|\delta g\|^{2}\right) .
$$

In other words, if the perturbations $\delta f$ and $\delta g$ are small, then the difference between $v$ and $u$ is small. It is interesting to note that the special case $\delta f=\delta g=0$ implies uniqueness in the linear case. We will as stated above not discuss existence in detail, but just note that it relies on imposing a minimal number of boundary conditions such that an energy estimate like (2.3) can be obtained. For more details on these fundamental aspects see $[5,6]$.

One of the reasons that energy estimates are so important in scientific computing is that small perturbations in the data is inevitable due to floatingpoint arithmetic and measurement errors. If such perturbations could cause unpredictable changes in the solution, then any numerical solution would be potentially unreliable. Hence, energy estimates like (2.3) provide a solid starting point for reliable computations.

One common way of arriving at an energy estimate is by using the energy method. The energy method typically proceeds as follows:

1. Multiply the PDE by the solution.

2. Integrate in space.

3. Note that $\int_{\Omega} u u_{t} d x=\int_{\Omega} \frac{1}{2}\left(u^{2}\right)_{t} d x=\frac{1}{2} \frac{d}{d t} \int_{\Omega} u^{2} d x=\frac{1}{2} \frac{d}{d t}\|u\|^{2}$. 
4. Manipulate the remaining integrals using integration by parts until only dissipative volume integrals and boundary integrals remain.

5. Insert boundary conditions that limit the boundary integrals.

6. Integrate in time.

Let us see what this means for the linear advection equation. We multiply (2.2) by $u$ :

$$
u u_{t}+u u_{x}=0 \Longleftrightarrow \frac{1}{2}\left(u^{2}\right)_{t}=-u u_{x} \Longleftrightarrow\left(u^{2}\right)_{t}=-2 u u_{x} .
$$

Next we integrate in space:

$$
\int_{0}^{1}\left(u^{2}\right)_{t} d x=-2 \int_{0}^{1} u u_{x} d x \Longleftrightarrow \frac{d}{d t}\|u\|^{2}=-2 \int_{0}^{1} u u_{x} d x .
$$

Using integration by parts we get that

$$
\int_{0}^{1} u u_{x} d x=\left.u^{2}\right|_{0} ^{1}-\int_{0}^{1} u_{x} u d x \Longleftrightarrow 2 \int_{0}^{1} u u_{x} d x=\left.u^{2}\right|_{0} ^{1} .
$$

Hence,

$$
\frac{d}{d t}\|u\|^{2}=\left.u^{2}\right|_{x=0}-\left.u^{2}\right|_{x=1} .
$$

The above relation is natural; we know that $u$ is flowing into the domain at the left boundary and out of the domain at the right boundary, resulting in the growth term $\left.u^{2}\right|_{x=0}$ at the left boundary, and the dissipative term $\left.u^{2}\right|_{x=1}$ at the right boundary. We do not get any dissipative integrals over the domain in this case, because the only way for a purely advective process to lose energy is by advection across the boundary. Dissipation inside the domain is typically the result of a diffusive process, which can be modelled by adding a second derivative term $\epsilon u_{x x}$ on the right-hand side of (2.2).

By inserting the boundary condition $\left.u\right|_{x=0}=g$ into (2.4), we get

$$
\frac{d}{d t}\|u\|^{2}=g^{2}-\left.u^{2}\right|_{x=1}
$$

Finally, integrating in time gives us the energy estimate:

$$
\|u(T)\|^{2} \leq\|f\|^{2}+\|g\|^{2}
$$


which is of the form (2.3) with $K=1$.

The above procedure is fairly straight forward, and in fact the energy analysis for most IBVPs follow the same pattern by identifying dissipation and growth at the boundary, and bounding the growth terms using boundary conditions. 



\section{Summation-by-parts operators}

The most obvious way of discretizing the spatial part of an IBVP is to use finite differences. This results in a system of ordinary differential equations (ODEs) that can be integrated in time using a standard ODE solver, for example a Runge-Kutta method. Summation-by-parts (SBP) operators were originally a class of matrices that relate to finite difference schemes that satisfy the SBP property - a discrete version of integration by parts $[7,8]$. Today SBP operators cover a wide range of discretization methods [9-13]. In this section we elucidate how the SBP property carries us part of the way to provable stability (i.e. a discrete energy estimate) of discretized IBVPs.

Before we formally define the SBP property, let us discuss some classical finite difference ideas. Consider an equidistant grid $x_{i}=i h, h=1 / N$, $i=0,1,2, \ldots, N$, and a differentiable function $u:[0,1] \rightarrow \mathbb{R}$. Let $u_{i}:=u\left(x_{i}\right)$ and $u_{i}^{\prime}=u^{\prime}\left(x_{i}\right)$. Recall that the derivative of $u$ can be approximated by finite differences. For example, second order accurate central differences may be used at interior points:

$$
u_{i}^{\prime}=\frac{u_{i+1}-u_{i-1}}{2 h}+\mathcal{O}\left(h^{2}\right),
$$

and first order accurate one sided differences may be used at boundary points:

$$
\begin{gathered}
u_{0}^{\prime}=\frac{u_{1}-u_{0}}{h}+\mathcal{O}(h), \\
u_{N}^{\prime}=\frac{u_{N}-u_{N-1}}{h}+\mathcal{O}(h) .
\end{gathered}
$$

We can write this compactly in matrix form:

$$
D \mathbf{u}=\mathbf{u}^{\prime}+\mathcal{O}(h),
$$


where

$$
D=\frac{1}{h}\left[\begin{array}{cccccc}
-1 & 1 & 0 & 0 & 0 & 0 \\
-1 / 2 & 0 & 1 / 2 & 0 & 0 & 0 \\
0 & -1 / 2 & 0 & 1 / 2 & 0 & 0 \\
0 & 0 & \ddots & \ddots & \ddots & \vdots \\
0 & 0 & \cdots & -1 / 2 & 0 & 1 / 2 \\
0 & 0 & \cdots & 0 & 1 & -1
\end{array}\right], \mathbf{u}=\left[\begin{array}{c}
u_{0} \\
u_{1} \\
\vdots \\
u_{N}
\end{array}\right], \mathbf{u}^{\prime}=\left[\begin{array}{c}
u_{0}^{\prime} \\
u_{1}^{\prime} \\
\vdots \\
u_{N}^{\prime}
\end{array}\right]
$$

and $\mathcal{O}(h)=\left[\begin{array}{lllll}\mathcal{O}(h) & \mathcal{O}\left(h^{2}\right) & \cdots & \mathcal{O}\left(h^{2}\right) & \mathcal{O}(h)\end{array}\right]^{\top}$.

Let us pause here for a moment and reflect on the fact that the matrix $D$ allows us to formulate a semi-discrete version of the linear advection equation (2.2):

$$
\mathbf{u}_{t}+D \mathbf{u}=\mathbf{0} .
$$

Of course, this formulation does not take the boundary condition into account, but let us return to that problem later. For now it looks like it should be possible to mimic at least part of the continuous energy analysis as long as $D$ satisfies some kind of integration by parts property. In fact, $D$ does satisfy such a property. Consider the quadrature matrix

$$
P=h\left[\begin{array}{llllll}
1 / 2 & & & & & \\
& 1 & & & & \\
& & 1 & & & \\
& & & \ddots & & \\
& & & & 1 & \\
& & & & & 1 / 2
\end{array}\right],
$$

and note that $P$ corresponds to the trapezoidal rule, i.e.,

$$
\mathbf{u}^{\top} P \mathbf{u}=\int_{0}^{1} u^{2} d x+O\left(h^{2}\right)
$$

Since $P$ is positive definite it also defines a norm $\|\mathbf{u}\|_{P}=\sqrt{\mathbf{u}^{\top} P \mathbf{u}} \approx\|u\|_{L^{2}}$. Let us use the matrix $P$ to mimic step 1 and 2 in the continuous energy analysis; multiplying by the solution and integrating in space. In other words, we multiply (3.1) by $\mathbf{u}^{\top} P$. This yields

$$
\mathbf{u}^{\top} P \mathbf{u}_{t}+\mathbf{u}^{\top} P D \mathbf{u}=\frac{1}{2} \frac{d}{d t}\|\mathbf{u}\|_{P}^{2}+\mathbf{u}^{\top} P D \mathbf{u}=0 .
$$


Note that $\mathbf{u}^{\top} P D \mathbf{u} \approx \int_{0}^{1} u u_{x} d x$ and recall that the next step in the continuous energy analysis was to perform integration by parts on the integral $\int_{0}^{1} u u_{x} d x$ in order to arrive at the identity $2 \int_{0}^{1} u u_{x} d x=\left.u\right|_{0} ^{1}$. To see that such a manipulation is possible also for the term $\mathbf{u}^{\top} P D \mathbf{u}$, let us take a closer look at the matrix $P D$ :

$$
P D=\left[\begin{array}{cccccc}
-1 / 2 & 1 / 2 & & & & \\
-1 / 2 & 0 & 1 / 2 & & & \\
& -1 / 2 & 0 & 1 / 2 & & \\
& & \ddots & \ddots & \ddots & \\
& & & -1 / 2 & 0 & 1 / 2 \\
& & & & -1 / 2 & 1 / 2
\end{array}\right]
$$

Note that $P D$ is almost skew-symmetric:

$$
P D+(P D)^{\top}=\left[\begin{array}{cccccc}
-1 & & & & & \\
& 0 & & & & \\
& & 0 & & & \\
& & & \ddots & & \\
& & & 0 & \\
& & & & & 1
\end{array}\right] .
$$

The only nonzero elements in (3.2) correspond to boundary terms, which suggests that we are on the right track. Indeed,

$$
\begin{aligned}
\mathbf{u}^{\top} P D \mathbf{u} & =\mathbf{u}^{\top}\left(P D+(P D)^{\top}-(P D)^{\top}\right) \mathbf{u} \\
& =\mathbf{u}^{\top}\left(P D+(P D)^{\top}\right) \mathbf{u}-\mathbf{u}^{\top}(P D)^{\top} \mathbf{u} \\
& =u_{N}^{2}-u_{0}^{2}-\mathbf{u}^{\top} D^{\top} P \mathbf{u} \\
& =u_{N}^{2}-u_{0}^{2}-(D \mathbf{u})^{\top} P \mathbf{u} \\
& =u_{N}^{2}-u_{0}^{2}-\mathbf{u}^{\top} P D \mathbf{u}
\end{aligned}
$$

implies that

$$
2 \mathbf{u}^{\top} P D \mathbf{u}=u_{N}^{2}-u_{0}^{2},
$$

which precisely corresponds to the identity $2 \int_{0}^{1} u u_{x} d x=\left.u\right|_{0} ^{1}$.

In the SBP literature, the matrix $P D$ is so important that it gets its own letter, $Q:=P D$, and in fact $Q+Q^{\top}=\operatorname{diag}(-1,0,0, \ldots, 1)$ is the SBP 
property. Returning to our discrete energy analysis we now have that

$$
\frac{1}{2} \frac{d}{d t}\|\mathbf{u}\|_{P}^{2}+\mathbf{u}^{\top} P D \mathbf{u}=0 \Longleftrightarrow \frac{d}{d t}\|\mathbf{u}\|_{P}^{2}=u_{0}^{2}-u_{N}^{2},
$$

in perfect correspondence with the continuous identity

$$
\frac{\partial}{\partial t}\|u\|^{2}=\left.u^{2}\right|_{x=0}-\left.u^{2}\right|_{x=1} .
$$

We summarize the section by a formal definition of the SBP property.

Definition 1. Let $P$ be a symmetric positive definite matrix. The matrix $D=P^{-1} Q$ is said to be an $S B P$ operator if

$$
Q+Q^{\top}=\operatorname{diag}(-1,0,0, \ldots, 1) .
$$

Definition 1 can be shown to hold for a large range of discretization matrices, including those used in higher order finite difference, discontinuous Galerkin, and finite volume methods.

It is straight forward to extend one-dimensional SBP operators to multiple dimensions by using Kronecker products. Consider an equidistant grid $\left\{\left(x_{i}, y_{j}\right)\right\}, x_{i}=i / N, y_{j}=j / N, i, j=0,1, \ldots, N$ (we use the same resolution in the $x$ and $y$ directions for simplicity, this is not necessary), on the unit square, and define

$$
\mathbf{D}_{x}:=D \otimes I, \quad \mathbf{D}_{y}:=I \otimes D,
$$

where $I$ is the $N \times N$ identity matrix and $D$ is an $N \times N$ SBP operator. The matrices $\mathbf{D}_{x}$ and $\mathbf{D}_{y}$ can be used to approximate the partial derivatives of a differentiable function $\phi=\phi(x, y)$. Indeed, if

$$
\boldsymbol{\phi}=\left[\begin{array}{llll}
\boldsymbol{\phi}_{0}^{\top} & \boldsymbol{\phi}_{1}^{\top} & \cdots & \boldsymbol{\phi}_{N}^{\top}
\end{array}\right]^{\top}, \quad \boldsymbol{\phi}_{i}=\left[\begin{array}{llll}
\phi\left(x_{i}, y_{0}\right) & \phi\left(x_{i}, y_{1}\right) & \cdots & \phi\left(x_{i}, y_{N}\right)
\end{array}\right]^{\top},
$$

then $\mathbf{D}_{x} \phi \approx \phi_{x}$ and $\mathbf{D}_{y} \phi \approx \phi_{y}$.

Remark 1. It is also possible to construct higher dimensional SBP operators without relying on one-dimensional operators, for example using finite volume or finite element methods $[14,15]$.

As mentioned earlier, the problem of imposing the boundary condition remains. A weak imposition of the boundary condition that complements the SBP property in a nice way will be described in the next section. 


\section{Weak boundary conditions}

The Simultaneous Approximation Term (SAT), introduced in [16] but originating from [17], is a penalty technique that imposes a boundary condition weakly. In other words, the numerical solution is allowed to deviate from the boundary condition, but any such deviation is penalized. We will show that as long as the solution does not satisfy the boundary condition, the SAT will add a contribution to the temporal derivative that corrects the deviation as we integrate in time.

Recall that the linear advection equation (2.2), together with the boundary condition $\left.u\right|_{x=0}=g$, implies that

$$
\frac{d}{d t}\|u\|^{2}=g^{2}-\left.u^{2}\right|_{x=1} .
$$

Furthermore, recall that the semi-discrete system (3.1) implies that

$$
\frac{d}{d t}\|\mathbf{u}\|_{P}^{2}=u_{0}^{2}-u_{N}^{2}
$$

We would like to adjust (3.1) to somehow account for the boundary condition and replace the growth term $u_{0}^{2}$ by $g^{2}$. To this end, consider the following SAT:

$$
\sigma P^{-1} \mathbf{e}_{0}\left(u_{0}-g\right)
$$

where $\sigma$ is a real parameter to be determined and $\mathbf{e}_{0}=\left[\begin{array}{lllll}1 & 0 & 0 & \cdots & 0\end{array}\right]^{\top}$. The idea is to add (4.1) to the right-hand side of (3.1). The presence of the matrix $P^{-1}$ in the SAT may appear strange, but it will become clear as we perform the stability analysis. Let us first note some desirable properties of the SAT:

- If the boundary condition is satisfied, the penalty vanishes. 
- If the boundary condition is not satisfied, the penalty adds a contribution to $d u_{0} / d t$.

- Multiplying the penalty by $\mathbf{u}^{\top} P$ (i.e. multiplying by the solution and integrating in space), results only in boundary terms.

Consider now a modified version of (3.1),

$$
\mathbf{u}_{t}+D \mathbf{u}=\sigma P^{-1} \mathbf{e}_{0}\left(u_{0}-g\right) .
$$

We can guess the sign of $\sigma$ by noting that if $u_{0}$ is greater than $g$, then the penalty should add a negative contribution to $\frac{d u_{0}}{d t}$. Conversely, if $u_{0}$ is less than $g$, then the penalty should add a positive contribution to $\frac{d u_{0}}{d t}$. Hence we should choose $\sigma<0$. Let us show that this is intuition is indeed correct (but not sufficient for stability).

Multiplying $\sigma P^{-1} \mathbf{e}_{0}\left(u_{0}-g\right)$ by $\mathbf{u}^{\top} P$ yields

$$
\mathbf{u}^{\top} P \sigma P^{-1} \mathbf{e}_{0}\left(u_{0}-g\right)=\sigma\left(u_{0}^{2}-u_{0} g\right) .
$$

Therefore,

$$
\frac{1}{2} \frac{d}{d t}\|\mathbf{u}\|_{P}^{2}+\mathbf{u}^{\top} P D \mathbf{u}=\sigma\left(u_{0}^{2}-u_{0} g\right)
$$

which leads to

$$
\begin{aligned}
\frac{d}{d t}\|\mathbf{u}\|_{P}^{2} & =u_{0}^{2}-u_{N}^{2}+2 \sigma\left(u_{0}^{2}-u_{0} g\right) \\
& =(2 \sigma+1) u_{0}^{2}-2 \sigma u_{0} g-u_{N}^{2} \\
& =(2 \sigma+1)\left[u_{0}^{2}-\frac{2 \sigma}{2 \sigma+1} u_{0} g\right]-u_{N}^{2} \\
& =(2 \sigma+1)\left[\left(u_{0}-\frac{\sigma}{2 \sigma+1} g\right)^{2}-\left(\frac{\sigma}{2 \sigma+1} g\right)^{2}\right]-u_{N}^{2} \\
& =(2 \sigma+1)\left(u_{0}-\frac{\sigma}{2 \sigma+1} g\right)^{2}-\frac{\sigma^{2}}{2 \sigma+1} g^{2}-u_{N}^{2} .
\end{aligned}
$$

The above calculation implies that for any $\sigma<-1 / 2$ we have

$$
\frac{d}{d t}\|\mathbf{u}\|_{P}^{2} \leq-\frac{\sigma^{2}}{2 \sigma+1} g^{2} .
$$


Consider for simplicity the case $\sigma=-1$. Integrating (4.3) in time gives us a discrete energy estimate,

$$
\|\mathbf{u}(T)\|_{P}^{2}-\|\mathbf{u}(0)\|_{P}^{2} \leq \int_{0}^{T} g^{2} d t \Longleftrightarrow\|\mathbf{u}(T)\|_{P}^{2} \leq\|g\|^{2}+\|\mathbf{f}\|_{P}^{2},
$$

where $\mathbf{f}$ is the initial data. Note that the discrete energy estimate perfectly mimics the continuous energy estimate (2.5). A discretization that implies an energy estimate is known as a stable discretization. A discrete energy estimate allows us to bound the energy of the numerical solution at any given time in terms of data.

Most of the articles in this thesis make use of so called multiblock schemes. In multiblock schemes, the domain $\Omega$ is split into subdomains, or blocks (usually in order to accommodate complex geometries). The blocks can be coupled using SATs, and a stability analysis similar to the one above is made to ensure that the couplings do not contribute to energy growth. 



\section{Artifical neural networks}

Machine learning is a broad category of theories, methods, and tools with a common purpose: to enable machines to solve tasks by analyzing data. It is a field that has seen explosive growth in recent decades due to a number of success stories, in particular in the fields of computer vision [18-20] and reinforcement learning $[21,22]$.

Articles IV and V of this thesis employ machine learning ideas and tools in order to aid traditional numerical methods for solving IBVPs. In particular we use the concept of an Artifical Neural Network (ANN). An ANN is a special kind of function from $\mathbb{R}^{n}$ to $\mathbb{R}^{m}$, inspired by biological neural networks. It is built out of layered, interconnected computational neurons (see Figure 5.1). To each neuron one associates a weight vector $\vec{w}$ representing the strengths of the connections to the neurons in the preceding layer, a real number $b$ representing the threshold voltage of the neuron (roughly the strength of the input needed for the neuron to activate), and an activation function $f: \mathbb{R} \rightarrow \mathbb{R}$. Each neuron outputs the value $f\left(\vec{w}^{\top} \vec{x}+b\right)$ to the neurons in the subsequent layer, where $\vec{x}$ is the input to the neuron. It can be shown that ANNs are dense in $L^{1}$, meaning that virtually any function can be approximated to arbitrary accuracy using an ANN [23]. There are many other function classes that are dense in $L^{1}$, that do not exhibit the same desirable properties with respect to pattern recognition. The reason why ANNs perform so well in a broad variety of problem settings is not fully understood, and a matter of ongoing research $[24,25]$.

Typically what we want to achieve with an ANN is to have it generalize correctly to unseen inputs given that it has been trained on a set of known inputs and outputs. For example, we may have a set of labelled images from $m$ categories (i.e. training data), and we want the network to correctly classify the images in the set and, crucially, also correctly classify images not 


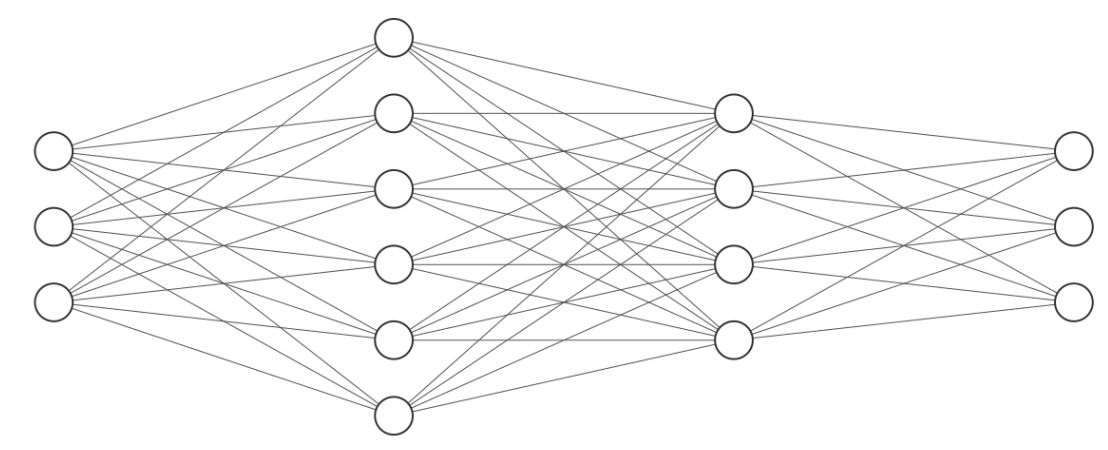

Input Layer $\in \mathbb{R}^{3}$

Hidden Layer $\in \mathbb{R}^{6}$

Hidden Layer $\in \mathbb{R}^{4}$

Output Layer $\in \mathbb{R}^{3}$

Figure 5.1: An artifical neural network with two hidden layers.

in the set. This is done by optimizing a loss function with respect to the network weights and biases. A loss function is a goodness of fit measure of the network with respect to the training data. ANNs are trained using gradient descent (or more commonly stochastic gradient descent) to minimize the loss function. The gradients are computed using a technique known as backpropagation [26]. To understand the idea, let us consider a very simple example, consisting of just four consecutive neurons. In the diagram below, the leftmost circle represents an input neuron. It simply passes the input $x^{*}$ to the first hidden neuron. At the first hidden neuron, the input is multiplied by the weight $w_{1}$. We denote this weighted input to the first hidden neuron by $z_{1}:=w_{1} x$. Then, the activation function $f=\tanh$ (this is a commonly used activation function since it is differentiable and approximates a Heaviside function) is applied to $z_{1}$, producing the output $y_{1}=\tanh \left(z_{1}\right)$. This output is passed on to the next neuron, and the process is repeated until the final neuron, the output neuron, is reached. The output neuron passes its output $y_{3}$ to the loss function $E\left(y_{3}, y^{*}\right)=\left(y_{3}-y^{*}\right)^{2}$, which quantifies the distance between the computed value $y_{3}$ and a known value $y^{*}$.

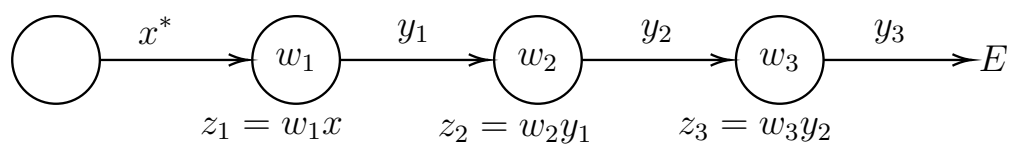


In order to minimize $E$ we adjust the weights $\left(w_{1}, w_{2}, w_{3}\right)$ in the direction of steepest descent, i.e. along the negative of the gradient of $E$. To compute the gradient $\left(\partial E / \partial w_{1}, \partial E / \partial w_{2}, \partial E / \partial w_{3}\right)$ given some data $\left(x^{*}, y^{*}\right)$, we start by evaluating the network at $x^{*}$ in order to compute $z_{1}, z_{2}, z_{3}$ and $y_{1}, y_{2}, y_{3}$ (this is known as a forward pass). Now note that by the chain rule we have

$$
\frac{\partial E}{\partial w_{i}}=\frac{\partial E}{\partial z_{i}} \frac{\partial z_{i}}{\partial w_{i}}=\frac{\partial E}{\partial z_{i}} y_{i-1}
$$

Here $y_{1}, y_{2}, y_{3}$ are known from the forward pass, so if we can compute $\partial E / \partial z_{1}$, $\partial E / \partial z_{2}$, and $\partial E / \partial z_{3}$, then we are done. It turns out that these derivatives can be computed inductively, starting from $\partial E / \partial z_{3}$ and working backwards (hence the name backpropagation). By the chain rule we get

$$
\frac{\partial E}{\partial z_{3}}=\frac{\partial E}{\partial y_{3}} \frac{\partial y_{3}}{\partial z_{3}}=2\left(y_{3}-y^{*}\right) f^{\prime}\left(z_{3}\right),
$$

where $f^{\prime}(z)=1-\tanh ^{2}(z)$. Furthermore, we have that

$$
\frac{\partial E}{\partial z_{i}}=\frac{\partial E}{\partial y_{i}} \frac{\partial y_{i}}{\partial z_{i}}=\frac{\partial E}{\partial z_{i+1}} \frac{\partial z_{i+1}}{\partial y_{i}} \frac{\partial y_{i}}{\partial z_{i}}=\frac{\partial E}{\partial z_{i+1}} w_{i+1} f^{\prime}\left(z_{i}\right) .
$$

That is, $\partial E / \partial z_{2}$ can be computed from $\partial E / \partial z_{3}$, and $\partial E / \partial z_{1}$ can be computed from $\partial E / \partial z_{2}$.

In practice, the above calculations are slightly more complex, since neurons will have more than one connection, but the principle is the same. In this thesis we have used the existing theory and concept of ANNs to improve certain aspects of numerical PDE calculations (see Article IV and Article V). 



\section{Summary of articles}

\subsection{Article I}

When we design a numerical scheme for solving an initial boundary value problem we typically start by discretizing in space. This results in a system of ordinary differential equations such as (4.2). Solving such a system is a nontrivial task in itself. A number of approaches exist, roughly divided into two major classes: explicit methods and implicit methods (though combinations, so called hybrid methods, also exist). Explicit methods advance the solution in time by applying an explicit operation to the current state of the system. In other words, if the solution $\mathbf{u}$ is known at time $t$, then an approximate solution at time $t+\Delta t$ can be found by operating explicitly on $\mathbf{u}(t)$. Implicit methods on the other hand, provides a relation between $\mathbf{u}(t)$ and $\mathbf{u}(t+\Delta t)$, and requires that a system of equations be solved in order to find $\mathbf{u}(t+\Delta t)$. Both methods have pros and cons. The explicit methods are easier, but restricted timesteps must be used for stability. The implicit methods often allow for unlimited timesteps but are much more complicated. As the resolution of the spatial discretization increases, the size of the system of equations increases, and the feasibility of solving the system with a computer decreases.

One class of implicit methods is found by discretizing in time using SBP operators $[27,28]$. Article I considers a multiblock discretization of a 2D advection-diffusion equation, based on SBP in space and time. The multiblock structure is leveraged to reduce the resulting system of equations to an equivalent set of smaller systems that can be solved more efficiently.

The idea can be illustrated by considering a two-block version of (4.2), discretized in time using SBP operators, where we solve for $\mathbf{u}$ on the subdomain $\Omega_{l}=[0,0.5]$ and for $\mathbf{v}$ on the subdomain $\Omega_{r}=[0.5,1]$. This yields the 
linear system

$$
\left[\begin{array}{cc}
A_{l} & \Sigma_{l} \\
\Sigma_{r} & A_{r}
\end{array}\right]\left[\begin{array}{l}
\mathbf{u} \\
\mathbf{v}
\end{array}\right]=\left[\begin{array}{l}
\mathbf{b}_{l} \\
\mathbf{b}_{r}
\end{array}\right]
$$

Here $A_{l}$ and $A_{r}$ contain the discretizations of the spatial and temporal derivatives, as well as the SATs enforcing the boundary condition at $x=0$, the initial condition at $t=0$, and the continuity condition $u=v$ at $x=0.5$. The off-diagonal blocks are sparse coupling matrices (nonzero only at elements corresponding to the interface node $x=0.5$ ) arising from the interface SATs. The right-hand side contains the initial and boundary data. One can show that $A_{l}$ and $A_{r}$ are non-singular, so that (6.1) is equivalent to

$$
\left[\begin{array}{cc}
I & A_{l}^{-1} \Sigma_{l} \\
A_{r}^{-1} \Sigma_{r} & I
\end{array}\right]\left[\begin{array}{l}
\mathbf{u} \\
\mathbf{v}
\end{array}\right]=\left[\begin{array}{l}
A_{l}^{-1} \mathbf{b}_{l} \\
A_{r}^{-1} \mathbf{b}_{r}
\end{array}\right]
$$

The construction of (6.2) involves solving a number of independent subdomain sized linear systems to compute the off-diagonal blocks and the new right-hand side. Furthermore, it can be shown that (6.2) can be reduced to a system involving only the interface elements. Once the interface system has been solved, (6.2) gives us the remaining elements as linear combinations of the interface elements. The idea can be seen by considering an explicit, but simplified version of (6.2):

$$
\left[\begin{array}{llll}
1 & 0 & a & 0 \\
0 & 1 & b & 0 \\
0 & c & 1 & 0 \\
0 & d & 0 & 1
\end{array}\right]\left[\begin{array}{l}
u_{0} \\
u_{1} \\
v_{0} \\
v_{1}
\end{array}\right]=\left[\begin{array}{l}
b_{0} \\
b_{1} \\
b_{2} \\
b_{3}
\end{array}\right] .
$$

In (6.3), $u_{1}$ and $v_{0}$ correspond to interface elements, which can be solved for using only the interior 2-by-2 part of the system:

$$
\left[\begin{array}{ll}
1 & b \\
c & 1
\end{array}\right]\left[\begin{array}{l}
u_{1} \\
v_{0}
\end{array}\right]=\left[\begin{array}{l}
b_{1} \\
b_{2}
\end{array}\right]
$$

Once $u_{1}$ and $v_{0}$ are known, (6.3) gives us explicit formulas for $u_{0}$ and $v_{1}$ :

$$
\begin{aligned}
& u_{0}=-a v_{0}+b_{0} \\
& v_{1}=-d u_{1}+b_{3} .
\end{aligned}
$$

Note that (6.1) is provably stable, so this procedure is a provably stable, non-iterative domain decomposition technique, as the title of the article suggests. 


\subsection{Article II}

A common way to handle curved domains is to introduce a mapping between the physical domain and a computational domain (usually the unit square or cube). The PDE is then restated on the computational domain using the chain rule, resulting in an equivalent PDE involving metric terms. This is done because standard SBP operators act on cartesian grids. The reformulation is often quite complicated, with lots of metric terms. In addition, the metric terms typically require a splitting procedure to ensure stability, introducing further complexity. Implementing such discretizations from scratch is error prone. In Article II we present a different perspective. We describe how the mapping between physical and computational space can be regarded as a stand alone operator construction. In other words, the switch to computational space can be built into so-called encapsulated SBP operators that act directly on curvilinear grids. These operators satisfy a curvilinear version of the summation-by-parts property (i.e. discrete versions of Green's identities). This approach has a number of benefits:

- Isolation of concerns. Discrete differentiation on a curvilinear grid is decoupled from PDE, and can therefore be implemented, debugged, and optimized in isolation.

- Clearer connection to the continuous problem.

- Simpler stability proofs, that often follow immediately from the cartesian case.

For example, consider the linear advection equation $u_{t}+a u_{x}+b u_{y}=0$ posed on a curved domain $\Omega$. A traditional discretization of this problem using SBP operators would look something like:

$$
\begin{aligned}
\mathbf{J} \mathbf{u}_{t}+\frac{1}{2} \mathbf{D}_{\xi}\left(a \mathbf{Y}_{\eta}\right. & \left.-b \mathbf{X}_{\eta}\right) \mathbf{u}+\frac{1}{2}\left(a \mathbf{Y}_{\eta}-b \mathbf{X}_{\eta}\right) \mathbf{D}_{\xi} \mathbf{u}+ \\
& +\frac{1}{2} \mathbf{D}_{\eta}\left(b \mathbf{X}_{\xi}-a \mathbf{Y}_{\xi}\right) \mathbf{u}+\frac{1}{2}\left(b \mathbf{X}_{\xi}-a \mathbf{Y}_{\xi}\right) \mathbf{D}_{\eta} \mathbf{u}=\mathrm{SAT}
\end{aligned}
$$

where $(\xi, \eta)$ are coordinates in computational space, and $\mathbf{D}_{\xi}$ and $\mathbf{D}_{\eta}$ are standard finite difference based SBP operators in the $\xi$ and $\eta$-directions respectively. The stability of this scheme is then proved using the energy method 
and the SBP properties of $\mathbf{D}_{\xi}$ and $\mathbf{D}_{\eta}$. Contrast this with the equivalent formulation

$$
\mathbf{u}_{t}+a \mathbf{D}_{x} \mathbf{u}+b \mathbf{D}_{y} \mathbf{u}=\mathrm{SAT},
$$

whose stability is also proved using the energy method and the (curvilinear) SBP properties of $\mathbf{D}_{x}$ and $\mathbf{D}_{y}$. The resulting proof has the added benefit of being much closer to how an energy estimate would be derived in the continuous setting (i.e. with no transformation of the domain). It should be stressed that (6.4) and (6.5) are completely equivalent. We have merely isolated the operators $\mathbf{D}_{x}$ and $\mathbf{D}_{y}$ in terms of $\mathbf{D}_{\xi}$ and $\mathbf{D}_{\eta}$, and found that they satisfy discrete versions of Green's identities. This removes the need to explicitly expose the mapping to computational space (such information hiding in favor of usability is precisely the concept of encapsulation from object oriented programming).

In Article I, a multiblock formulation was used to increase efficiency by exploiting the system structure. Multiblock formulations can also impact efficiency because the grid resolution can be changed from one block to the next. However, extra care must be taken to ensure the stability of such schemes, because the coupling of non-conforming grids requires interpolation. This problem has essentially been solved in earlier work [29,30] (though questionmarks for curved interfaces remain). In article II we additionally show how encapsulated SBP operators can be used in conjunction with the work in [29] to reduce the stability analysis of non-conforming couplings to the conforming case.

\subsection{Article III}

When computing approximate solutions to PDEs we are sometimes interested in preserving certain invariances of the exact solution. For example, in incompressible flow simulations we may want to make sure that our approximation is divergence free. Guaranteeing that such invariances hold exactly for an approximation often requires special consideration in the discretization design. In Article III we consider the Lindblad equation describing the evolution of dissipative quantum systems, and in particular its trace preserving property

$$
\int \delta(x-y) \rho d x d y \equiv 1,
$$

where $\rho$ is a reduced density operator. 
Trace preservation cannot be guaranteed to hold exactly in a standard SBP-SAT discretization of the Lindblad equation, because the derivation of (6.6) relies (in addition to integration by parts) on a property that does not hold discretely. More precisely, consider the change of variables $z=x-y$ and $z^{\prime}=x+y$. Then the partial derivatives $\frac{\partial}{\partial x}, \frac{\partial}{\partial y}, \frac{\partial}{\partial z}, \frac{\partial}{\partial z^{\prime}}$ are related by the equations

$$
\frac{\partial}{\partial z}=\frac{1}{2}\left(\frac{\partial}{\partial x}-\frac{\partial}{\partial y}\right), \quad \frac{\partial}{\partial z^{\prime}}=\frac{1}{2}\left(\frac{\partial}{\partial x}+\frac{\partial}{\partial y}\right) .
$$

Note that for functions $\phi=\phi(z)$ and $\psi=\psi\left(z^{\prime}\right)$ we have

$$
\frac{\partial}{\partial z}(\phi \psi)=\psi \frac{\partial}{\partial z} \phi, \quad \frac{\partial}{\partial z^{\prime}}(\phi \psi)=\phi \frac{\partial}{\partial z^{\prime}} \psi .
$$

The relations in (6.7) and (6.8) are necessary in order to show that the trace invariance (6.6) follows from the Lindblad equation. However, the naive discretizations of the operators $\partial / \partial z$ and $\partial / \partial z^{\prime}$ as

$$
\mathbf{D}_{z}=\left(\mathbf{D}_{x}-\mathbf{D}_{y}\right) / 2, \quad \mathbf{D}_{z^{\prime}}=\left(\mathbf{D}_{x}+\mathbf{D}_{y}\right) / 2
$$

using conventional finite difference based SBP operators $\mathbf{D}_{x}$ and $\mathbf{D}_{y}$, do not satisfy (6.8). In order to satisfy (6.8) while maintaining the SBP property, one can consider an averaging of periodic SBP operators based on second order central differences. That is, instead of the usual definition (3.3) of $\mathbf{D}_{x}$ and $\mathbf{D}_{y}$, we use

$$
\mathbf{D}_{x}:=\frac{1}{2}\left(D \otimes S_{+}+D \otimes S_{-}\right), \quad \mathbf{D}_{y}:=\frac{1}{2}\left(S_{+} \otimes D+S_{-} \otimes D\right),
$$

where $D$ is a second order periodic central difference operator and $S_{+}$and $S_{-}$are shift operators:

$$
S_{+}=\left[\begin{array}{cccccc}
0 & 0 & 0 & \cdots & 0 & 1 \\
1 & 0 & 0 & & 0 & 0 \\
0 & 1 & 0 & & 0 & 0 \\
0 & 0 & 1 & & 0 & 0 \\
\vdots & \vdots & & \ddots & \vdots & \vdots \\
0 & 0 & \cdots & 0 & 1 & 0
\end{array}\right], \quad S_{-}=S_{+}^{\top} .
$$

In Article III we show that the operators $\mathbf{D}_{z}, \mathbf{D}_{z^{\prime}}$ do satisfy (6.8) if they are built using the averaged operators in (6.10). We also show that they can be used to discretize the Lindblad equations such that the trace of the numerical solution is preserved. 


\subsection{Article IV}

When a viscous fluid flows along a surface, a so called boundary layer forms. At the surface, the flow velocity vanishes, causing very steep velocity gradients close to the surface for fast moving fluids. Boundary layers are notoriously difficult to capture efficiently and accurately with numerical schemes because of the large number of grid points needed to resolve them. Underresolved boundary layers tend to cause numerical oscillations that propagate throughout the domain. One way to counteract such oscillations is the use a procedure called the Multiple Penalty Technique (MPT) [31]. The idea is simple: impose known data in the interior of the domain using penalty terms (SATs). Of course, an obvious problem arises - from where do we get this data? After all, the point of the simulation is to produce such data. One source of data is experimental measurements. On the other hand, if we had access to experimental measurements, then why do we need the simulation? Another source of data is obtained by using high resolution simulations, which is the very procedure we are trying to avoid. Suppose, however, that we have access to such data (either through experiments or highly resolved simulations) merely for a limited set of fluid paramaters (viscosity, free stream velocity, angle of attack, etc). Can we leverage this information in order to run coarse grid simulations using fluid paramaters for which we do not have interior data? In Article IV we train a neural network to predict a boundary layer as a function of a diffusion parameter. The network is trained on data from a small number of highly resolved simulations with varying diffusion parameters, and used to predict the boundary layer for arbitrary diffusion parameters. The procedure works well, is provably stable, and the result of a coarse grid simulation aided by neural network predictions and MPT can be seen in Figure 6.1.

\subsection{Article V}

Meshing (grid generation) is a major aspect of PDE discretization, and is virtually a science of its own. Whether it is quadrilateral structured grids as in the case of finite difference methods, or triangular unstructured grids as in the case of finite element methods, some type of elaborate mesh generation appears unavoidable. One of the long term goals of the work in Article V is to reduce or eliminate the need for complex mesh generation, and ultimately 

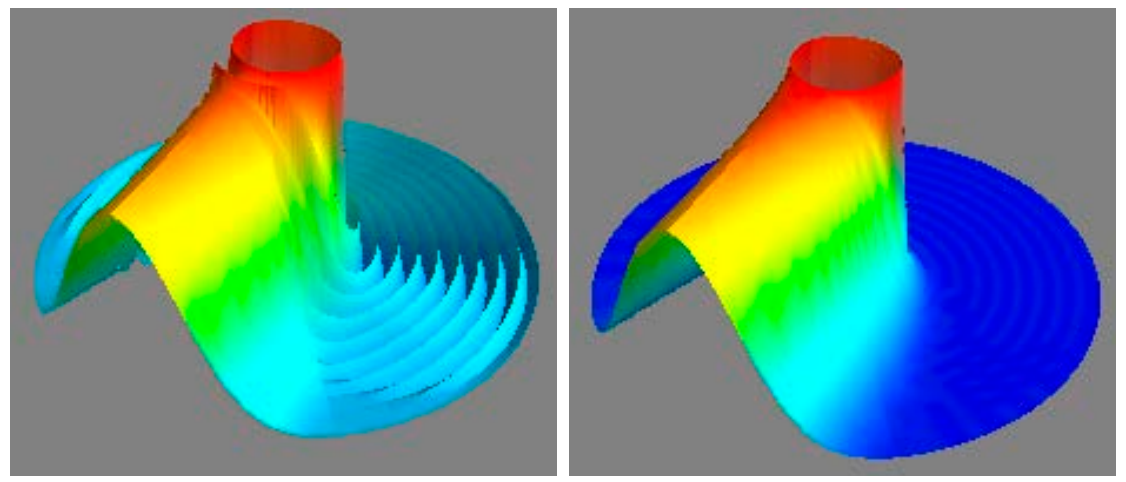

Figure 6.1: Left: A standard coarse grid simulation of a boundary layer, resulting in numerical oscillations. Right: A coarse grid simulation of a boundary layer, aided by neural network predictions and the multiple penalty technique.

rely solely on very general point clouds. Similar ambitions have been explored in the literature by training an ANN to solve a PDE at an arbitrary set of points, using the ANN as a solution ansatz [32]. In contrast, Article V uses a combination of traditional discretization concepts such as SBP-SAT together with ideas and tools from machine learning and optimization in order to produce discrete differentiation operators on an arbitrary set of points.

Consider an element $[a, b]$ with an arbitrary nodal distribution $a=x_{0}<$ $x_{1}<\cdots<x_{n}=b$ (the endpoints are included for simplicity). The problem of finding a discrete differentiation operator $D$ on this element can be formulated as a system of linear equations. Given a polynomial basis $\phi_{0}, \phi_{1}, \ldots, \phi_{q}$ we want to find $D$ such that

$$
D \Phi=\Phi^{\prime},
$$

where

$\Phi=\left[\begin{array}{cccc}\phi_{0}\left(x_{0}\right) & \phi_{1}\left(x_{0}\right) & \cdots & \phi_{q}\left(x_{0}\right) \\ \phi_{0}\left(x_{1}\right) & \phi_{1}\left(x_{1}\right) & \cdots & \phi_{q}\left(x_{1}\right) \\ \vdots & \vdots & \cdots & \vdots \\ \phi_{0}\left(x_{n}\right) & \phi_{1}\left(x_{n}\right) & \cdots & \phi_{q}\left(x_{n}\right)\end{array}\right], \Phi^{\prime}=\left[\begin{array}{cccc}\phi_{0}^{\prime}\left(x_{0}\right) & \phi_{1}^{\prime}\left(x_{0}\right) & \cdots & \phi_{q}^{\prime}\left(x_{0}\right) \\ \phi_{0}^{\prime}\left(x_{1}\right) & \phi_{1}^{\prime}\left(x_{1}\right) & \cdots & \phi_{q}^{\prime}\left(x_{1}\right) \\ \vdots & \vdots & \cdots & \vdots \\ \phi_{0}^{\prime}\left(x_{n}\right) & \phi_{1}^{\prime}\left(x_{n}\right) & \cdots & \phi_{q}^{\prime}\left(x_{n}\right)\end{array}\right]$.

However, such an operator is not well suited for use in IBVPs, because it does not generally lead to stability. We would like an additional property 
to hold for $D$, namely the SBP property. Recall from definition 1 that this means that $D=P^{-1} Q$, where $P$ is a positive definite quadrature matrix and $Q+Q^{\top}=\operatorname{diag}(-1,0,0, \ldots, 1)$. Thus we may instead try to first find positive quadrature weights $p_{0}, p_{1}, \ldots, p_{n}$ such that

$$
\left[\begin{array}{llll}
p_{0} & p_{1} & \cdots & p_{n}
\end{array}\right] \Phi=\left[\begin{array}{llll}
\int_{a}^{b} \phi_{0} d x & \int_{a}^{b} \phi_{1} d x & \cdots & \int_{a}^{b} \phi_{2 q} d x
\end{array}\right],
$$

followed by a $Q$ such that $D=P^{-1} Q$ satisfies (6.11) and such that $Q+Q^{\top}=$ $\operatorname{diag}(-1,0,0, \ldots, 1)$. Both finding $P$ and $Q$ are optimization problems that fit nicely into the machine learning paradigm. In Article $\mathrm{V}$ we use the machine learning library TensorFlow to compute operators like this in order to solve a one-dimensional model problem on overlapping grids.

While TensorFlow does not outperform traditional computational software (like Matlab or SciPy) in terms of efficiency in this case, it does allow for a significantly simpler implementation, something that we foresee as a growing advantage in future research of higher dimensional cases. 


\section{References}

[1] Erwin Schrödinger. An undulatory theory of the mechanics of atoms and molecules. Physical review, 28(6):1049, 1926.

[2] George Gabriel Stokes et al. On the effect of the internal friction of fluids on the motion of pendulums. 1851.

[3] Daniele Galli, Francesco Palla, Federico Ferrini, and O Straniero. Galactic evolution of $\mathrm{d}$ and 3 he. In The Light Element Abundances, pages 224-227. Springer, 1995.

[4] Charles L Fefferman. Existence and smoothness of the Navier-Stokes equation. The millennium prize problems, 57:67, 2006.

[5] B. Gustafsson, H.O. Kreiss, and J. Oliger. Time Dependent Problems and Difference Methods. A Wiley-Interscience Publication. Wiley, 1995.

[6] H.O. Kreiss and J. Lorenz. Initial-Boundary Problems and the NavierStokes Equation. Classics in Applied Mathematics. Society for Industrial and Applied Mathematics, 2004.

[7] H-O Kreiss and Godela Scherer. Finite element and finite difference methods for hyperbolic partial differential equations. In Mathematical aspects of finite elements in partial differential equations, pages 195-212. Elsevier, 1974.

[8] Bo Strand. Summation by parts for finite difference approximations for d/dx. Journal of Computational Physics, 110(1):47-67, 1994.

[9] Mark H Carpenter and David Gottlieb. Spectral methods on arbitrary grids. Journal of Computational Physics, 129(1):74-86, 1996. 
[10] Gregor J Gassner. A skew-symmetric discontinuous Galerkin spectral element discretization and its relation to SBP-SAT finite difference methods. SIAM Journal on Scientific Computing, 35(3):A1233-A1253, 2013.

[11] Ken Mattsson, Frank Ham, and Gianluca Iaccarino. Stable and accurate wave-propagation in discontinuous media. Journal of Computational Physics, 227(19):8753-8767, 2008.

[12] Jan Nordström, Karl Forsberg, Carl Adamsson, and Peter Eliasson. Finite volume methods, unstructured meshes and strict stability for hyperbolic problems. Applied Numerical Mathematics, 45(4):453-473, 2003.

[13] Hendrik Ranocha, Philipp Öffner, and Thomas Sonar. Summation-byparts operators for correction procedureia reconstruction. Journal of Computational Physics, 311:299-328, 2016.

[14] Magnus Svärd and Jan Nordström. Stability of finite volume approximations for the Laplacian operator on quadrilateral and triangular grids. Applied Numerical Mathematics, 51(1):101-125, 2004.

[15] Rémi Abgrall, Jan Nordström, Philipp Öffner, and Svetlana Tokareva. Analysis of the SBP-SAT stabilization for finite element methods part I: Linear problems. Journal of Scientific Computing, 85(2):1-29, 2020.

[16] Mark H Carpenter, David Gottlieb, and Saul Abarbanel. Time-stable boundary conditions for finite-difference schemes solving hyperbolic systems: methodology and application to high-order compact schemes. Journal of Computational Physics, 111(2):220-236, 1994.

[17] Daniele Funaro and David Gottlieb. A new method of imposing boundary conditions in pseudospectral approximations of hyperbolic equations. Mathematics of computation, 51(184):599-613, 1988.

[18] Alex Krizhevsky, Ilya Sutskever, and Geoffrey E Hinton. Imagenet classification with deep convolutional neural networks. In Advances in neural information processing systems, pages 1097-1105, 2012.

[19] Ross Girshick, Jeff Donahue, Trevor Darrell, and Jitendra Malik. Rich feature hierarchies for accurate object detection and semantic segmentation. In Proceedings of the IEEE conference on computerision and pattern recognition, pages 580-587, 2014. 
[20] Wei Liu, Dragomir Anguelov, Dumitru Erhan, Christian Szegedy, Scott Reed, Cheng-Yang Fu, and Alexander C Berg. Ssd: Single shot multibox detector. In European conference on computerision, pages 21-37. Springer, 2016.

[21] Volodymyr Mnih, Koray Kavukcuoglu, David Silver, Alex Graves, Ioannis Antonoglou, Daan Wierstra, and Martin Riedmiller. Playing atari with deep reinforcement learning. arXiv preprint arXiv:1312.5602, 2013.

[22] David Silver, Aja Huang, Chris J Maddison, Arthur Guez, Laurent Sifre, George Van Den Driessche, Julian Schrittwieser, Ioannis Antonoglou, Veda Panneershelvam, Marc Lanctot, et al. Mastering the game of go with deep neural networks and tree search. nature, 529(7587):484-489, 2016.

[23] Zhou Lu, Hongming Pu, Feicheng Wang, Zhiqiang Hu, and Liwei Wang. The expressive power of neural networks: Aiew from the width. In Advances in neural information processing systems, pages 6231-6239, 2017.

[24] Naftali Tishby and Noga Zaslavsky. Deep learning and the information bottleneck principle. In 2015 IEEE Information Theory Workshop (ITW), pages 1-5. IEEE, 2015.

[25] Henry W Lin, Max Tegmark, and David Rolnick. Why does deep and cheap learning work so well? Journal of Statistical Physics, 168(6):12231247, 2017.

[26] David E Rumelhart, Geoffrey E Hinton, and Ronald J Williams. Learning representations by back-propagating errors. nature, 323(6088):533536, 1986.

[27] Jan Nordström and Tomas Lundquist. Summation-by-parts in time. Journal of Computational Physics, 251:487-499, 2013.

[28] Tomas Lundquist and Jan Nordström. The SBP-SAT technique for initial value problems. Journal of Computational Physics, 270:86-104, 2014 . 
[29] Jeremy E Kozdon and Lucas C Wilcox. Stable coupling of nonconforming, high-order finite difference methods. SIAM Journal on Scientific Computing, 38(2):A923-A952, 2016.

[30] Ken Mattsson and Mark H Carpenter. Stable and accurate interpolation operators for high-order multiblock finite difference methods. SIAM Journal on Scientific Computing, 32(4):2298-2320, 2010.

[31] Jan Nordström, Qaisar Abbas, Brittany A Erickson, and Hannes Frenander. A flexible boundary procedure for hyperbolic problems: multiple penalty terms applied in a domain. Communications in Computational Physics, 16(2):541-570, 2014.

[32] Jens Berg and Kaj Nyström. A unified deep artificial neural network approach to partial differential equations in complex geometries. Neurocomputing, 317:28-41, 2018. 


\section{Papers}

The papers associated with this thesis have been removed for copyright reasons. For more details about these see:

http://urn.kb.se/resolve?urn=urn:nbn:se:liu:diva-171230 



\section{FACULTY OF SCIENCE AND ENGINEERING}

Linköping Studies in Science and Technology, Dissertation No. 2106, 2020 Department of Mathematics

Linköping University

SE-581 83 Linköping, Sweden

www.liu.se 